\title{
Intermittent 20-HZ-photic stimulation leads to a uniform reduction of alpha-Global Field Power in healthy volunteers
}

\author{
R. Rau ${ }^{1}$, C. Raschka ${ }^{2}$, H. J. Koch ${ }^{3}$ \\ ${ }^{1}$ Public Health Department, Regensburg, Germany \\ ${ }^{2}$ Institute of Sportsmedicine, J.-W.-Goethe-University, Regensburg, Germany \\ ${ }^{3}$ Dept. of Gerontopsychiatry, University Clinic of Psychiatry, Regensburg, Germany
}

Received: April 18, 2000

Accepted: April 21, 2001

19-channel-EEGs were recorded from scalp surface of 30 healthy subjects $(16 \mathrm{~m}, 14 \mathrm{f}$, mean age: 34 ys, SD: $11.7 \mathrm{ys}$ ) at rest and under IPS (Intermittent Photic Stimulation) at rates of 5, 10 and $20 \mathrm{Hertz}(\mathrm{Hz})$. Digitalized data underwent spectral analysis with fast fourier transformation (FFT) yielding the basis for the computation of global field power (GFP). For quantification GFP values in the frequency ranges of 5,10 and $20 \mathrm{~Hz}$ at rest were divided by the corresponding data gained under IPS. While ratios from PDE data showed no stable parameter due to high interindividual variability, ratios of alpha-power turned out to be uniform in all subjects: IPS at $20 \mathrm{~Hz}$ always led to a suppression of alpha-power. Dividing alpha-GFP at rest by alpha-GFP under $20-\mathrm{Hz}$ IPS thus resulted in a ratio $<1$. We conclude that ratios from GFP data are a stable diagnostic paradigma. ratios

Keywords: EEG, brain mapping, intermittent photic stimulation (IPS), global field power

First studies on alterations of electric cerebral activity induced by Intermittent Photic Stimulation (IPS) were done by Adrian and Matthews in 1934 (19). It was observed that brain waves can be synchronised by external stimulation with rhythmic flashlights, the so-called photic driving effect (PDE). In this physiological reaction interindividual variability is high; in some cases coupling to stimulation rates does only

Correspondence should be addressed to

Horst Koch, MD, PhD, MFPM, DCPSA

Department of Gerontopsychiatry

University Clinic of Psychiatry

D-93053 Regensburg, Universitätsstrasse 84, Deutschland

Phone: + 49-941-941-0

Fax: + 49-941-941-1205 
or most visibly occur at frequencies corresponding to the spontaneous rhythm ("frequency selectivity") $(12,19)$.

GFP is nowadays a widely used technique in clinical neurophysiology such as visual discrimation performance during saccades or epilepsy research $(2,14)$. GFP analysis can serve to monitor treatment of Alzheimer's disease with tacrin or related drugs $(7,8)$. In cerebrovascular diseases an increase of PDE was observed (19). Alterations of PDE were described in migraine patients and other forms of headache (10). Apart from the PDE, the phenomenon of alpha-suppression had been explored by several investigators, reporting differences in headache and migraine patients: IPS at rates of $20 \mathrm{~Hz}$ in patients suffering from migraine did not lead to alpha-suppression, which occurs in healthy persons; in some cases, IPS elicited even an activation of brain waves similar to $\operatorname{PDE}(5,6,13,15,16)$.

Heretofore, data from single electrodes are used to calculate power. The objective of this study was to assess whether a decrease of GFP as a measure of overall EEG power, instead of power data from single electrodes, can serve as a useful experimental or diagnostic paradigma.

\section{Materials and Methods}

EEGs of 30 healthy volunteers ( 16 male and 14 female; mean age $=34 \mathrm{ys,}$ $\mathrm{sd}=11.7 \mathrm{ys})$ were recorded at rest for at least $30 \mathrm{~s}$ and under IPS-series of $30 \mathrm{~s}$ each at 5, 10 and $20 \mathrm{~Hz}$. Flashlight stimulation was applied from a stroboscope placed at a distance of $20 \mathrm{~cm}$ in front of the subjects who closed their eyes. Each train of flashlight stimulation was followed by an interval of $20 \mathrm{~s}$. Recordings were performed under standardized conditions.

EEG were recorded with 19 surface electrodes, arranged according to the international ten-twenty-system, with a time constant of $0.3 \mathrm{~s}$ and a lowpass filter of $70 \mathrm{~Hz}$. Frequency of digitalization was $512 \mathrm{~Hz}$. Data were digitalized and computed with Fast Fourier Transformation (FFT) for spectral analysis and brain mapping representation (3). The GFP is a reference-free measure of the variability of potentials calculated from EEG data by interpolation $(9,11,19)$.

From each phase of EEGs 20 seconds were gained for spectral analysis. Artifacts, due to muscle contraction, twinkling, transpiration, etc., were excluded in the off-line analysis. Data analysis focussed on four frequency bands: firstly ranges were defined according to the stimulation frequencies $\pm 10 \%$. That is, the $5 \mathrm{~Hz}$-frequency range went from 4.5 to $5.5 \mathrm{~Hz}$; the same procedure was applied to the $10 \mathrm{~Hz}$ and $20 \mathrm{~Hz}$ range. Alpha range was confined to a range of 8 to $12 \mathrm{~Hz}$. 
The GFP-data of 30 healthy volunteers at rest were compared to those gained during IPS at 5, 10 and $20 \mathrm{~Hz}$. In order to quantify PDE, ratios were computed from GFP-data at rest in comparison with those obtained from EEGs under IPS. All data were analysed descriptively. Parametric $t$-tests for related samples were used and $t$-value maps were calculated (4). Nonparametric Wilcoxon tests for related samples were applied for comparison of alpha-GFP-values at rest and under stimulation (17).

\section{Results}

All subjects showed normal EEG results, which can be allocated to classical categories. PDE was observed in all subjects. GFP at rest and under IPS displayed a high interindividual variability within the defined frequency ranges, yet data showed normal distribution. Due to this variable response to light flashes no stable ratio of GFP values could be calculated for 5, 10 and $20 \mathrm{~Hz}$ range during stimulation with 5, 10 and $20 \mathrm{~Hz}$, respectively.

On the contrary, ratios from alpha-power yielded more uniform results: while activity under $10 \mathrm{~Hz}$-IPS produced inconsistent changes with reduction of alpha-power in approximately $70 \%$ of the subjects, IPS with 20 flashes per second led in all cases to a reduction of alpha-GFP. The distribution of ratios of alpha-GFP under $20-\mathrm{Hz}$ IPS by alpha-GFP at rest resulted in values <1 (Fig. 1). GFP differences between values at rest and under IPS were highly significant $(\mathrm{p}<0.001)$. T-test analysis of spectral data above single electrodes revealed that power reduction was most pronounced in the parietooccipital region.

\section{Discussion}

In contrast to the results of previous studies $(15,19)$ all 30 volunteers in the present examination showed PDE with a high interindividual variability. Even in EEG samples with only mild PDE, spectral analysis rendered visible peaks of electric field power in the frequency range that corresponded to the stimulation rate and partially revealed harmonics. In accordance with earlier studies (6) reporting suppression by photic and acoustic stimulation, we found a reduction of GFP in the $10 \mathrm{~Hz}$-domain under IPS in the majority of cases (70\%). 


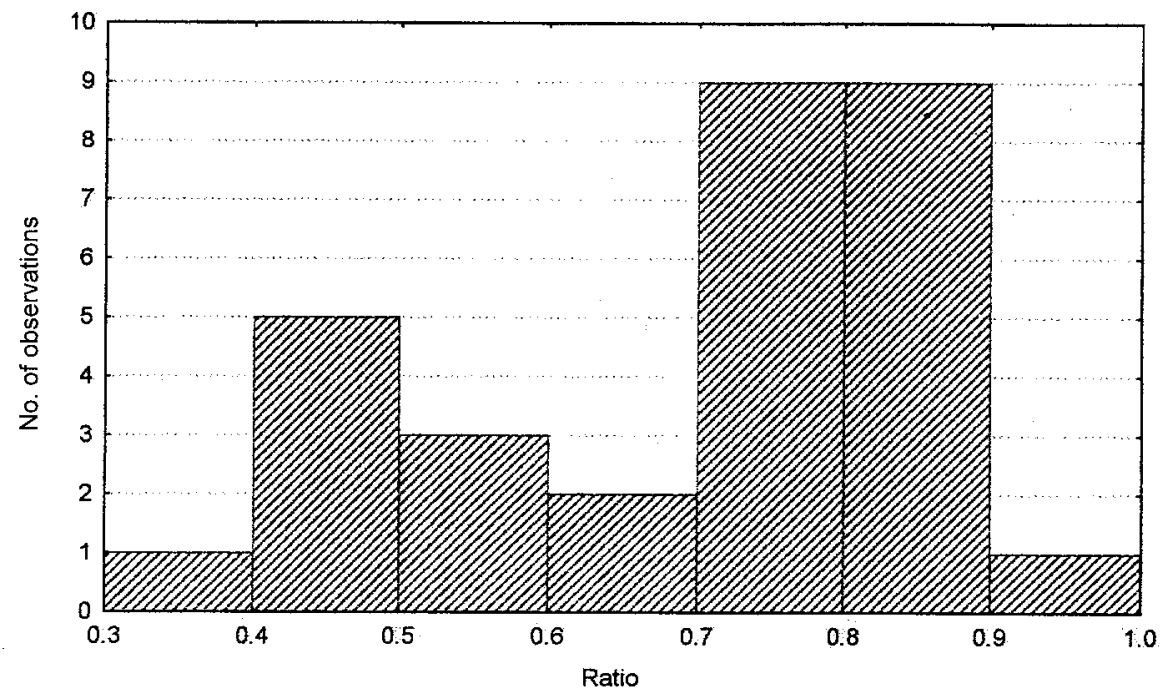

Fig. 1. Distribution of ratios of Alpha-GFP following IPS with $20 \mathrm{~Hz}$ and at rest in 30 healthy volunteers

As the essential result of our study under the influence of $20-\mathrm{Hz}$ IPS GFP decreased below the initial level (GFP at rest) in almost all cases. The calculated GFPratio proved to be a stable characteristic beeing smaller than one in all subjects. Previous investigations in healthy persons and headache or migraine patients compared local EEG-changes above single electrodes $(5,13,16)$. These studies revealed a decrease of power at single occipito-parietal electrodes induced by $20-\mathrm{Hz}$ IPS in normal healthy volunteers.

In the present study, however, attenuation of alpha-power led to a uniform decrease of GFP in the alpha-range compared to the initial data, which indicates general desynchronisation of brain acitivity. So, it was possible to form an $\alpha$-GFP-quotient, calculated on the basis of data at rest and data under $20-\mathrm{Hz}$ IPS, which can be considered as a stable parameter in EEG diagnosis. In this study, all healthy subjects showed a quotient $<1$. We conclude that GFP as a global and general parameter does still reflect local changes induced by IPS in the alpha-range reliably and may thus be a practical instrument in daily clinical work. Further investigation will be needed to gather comparable ratios from patients' EEG data. In this context, migraine and headache patients will be of special interest, since previous studies along with preliminary findings in our clinical experience suggest that migraine patients show significantly different electrophysiologic patterns of reaction to IPS with flashlight in comparison to healthy controls $(5,13,16)$. 


\section{REFERENCES}

1. American Electroencephalographic Society. Statement on the clinical use of quantitative EEG. J. Clin. Neurophysiol. 4, 197 (1987).

2. Anagnoustou, E., Kleiser, R., Skrandies, W.: Electrophysiological correlates of human intrasaccadic processing. Exp. Brain Res. 130, 177-187 (2000)

3. Brandl, U., Wenzel, D. (1989): Advanced programming techniques for activity mapping on personal computers. In: Topographic brain mapping of EEG and evoked potentials. Ed.: Maurer, K. Springer, Heidelberg, pp. 129-135.

4. Duffy, F. H. (1989): Topographic mapping of brain electrical activity: Clinical applications and issues. In: Topographic brain mapping of EEG and evoked potentials Ed.: Maurer, K, Springer, Heidelberg, 19-52.

5. Genco, S., de Tommaso, M., Prudenzano, A. M., Savarese, M., Puca, F. M.: EEG features in juvenile migraine: topographic analysis of spontaneous and visual evoked brain electrical activity: a comparison with adult migraine. Cephalgia 14, 41-6 (1994).

6. Grillon, C., Buchsbaum, M. S.: Computed EEG-Topography of response to visual and auditory stimuli. Electrenc. Clin. Neurophysiol. 63, 42-53 (1986).

7. Jelic, V., Dierks, T., Amberla, K., Almkvist, O., Winblad, B., Nordberg, A.: Longitudinal changes in quantitative EEG during long-term tacrine treatment of patients with Alzheimer's disease. Neurosci. Lett. 254, 85-88 (1998).

8. Jelic, V., Blomberg, M., Dierks, T., Basun, H., Shigeta, M., Julin, P., Jensen, M., Lannfelt, L., Winblad, B., Wahlund, L. O.: EEG slowing and cerebrospinal fluid tau levels in patients with cognitive decline. Neuroreport 9 157-160 (1998).

9. Klotz, J. M.: Topographic EEG mapping methods. Cephalgia 13, 45-52 (1993).

10. Klotz, J. M., Heils, A., Langohr, H. D.: Quantitative und topographische Analyse des Photic-DrivingEffektes bei Patienten mit Migraine. Nervenheilkunde 11, 316-322 (1992).

11. Lehmann, D., Skrandies, W.: Reference-free identification of components of checker-board-evoked, multi-channel-potential-field. Electroenc. Clin. Neurophysiol. 48, 609-621 (1980).

12. Mast, J., Victor, J. D.: Fluctuations of steady-state VEPs: interaction of driven evoked potentials and the EEG. Electroenc. Clin. Neurophysiol. 78, 389-401 (1991).

13. Puca, F. M., de Tommaso, Tota, P., Sciruicchio, V.: Photic driving in migraine: correlations with clinical features. Cephalalgia 16, 246-50 (1996).

14. Skrandies, W., Reik, P., Kunze, C.: Topography of evoked brain activity during mental arithmetic and language tasks: sex differences. Neuropsychologia 37, 421-430 (1999).

15. Takahashi, T. (1993): Activation methods. In: Electroencephalography. 3rd edition, Eds: Niedermeyer, E., Lopes da Silva, F., Williams \& Wilkins, Baltimore, pp. 241-62.

16. Tsounis, S., Varfis, G.: Alpha rhythm power and the effect of photic stimulation in migraine with brain mapping. Clin. Electroenc. 23, 1-6 (1992).

17. Zar, J. H.: Biostatistical Analysis. Prentice Hall International Edition, Englewood Cliffs (1984).

18. Zwiener, U., Eiselt, M., Flemming, L., Wagner, H., Schack, B.: Early magnetic field changes preceding the intracortical penicillin induced spikes. Epilepsy Res. 38, 217-229 (2000).

19. Zschocke, S. (1995): Klinische Elektroenzephalographie, Springer, pp. 211-221. 